\title{
RADIOLOGICAL AND CLINICAL ASSESSMENT OF TWO GROUPS OF ANCHORS IN DOUBLE-ROW ROTATOR CUFF REPAIR
}

\author{
Ojas Saini' ${ }^{1}$ Rajendra Saini², Umesh Kumar Meena ${ }^{3}$, Tejas Patel ${ }^{4}$, Vishal Singh ${ }^{5}$ \\ ${ }^{1}$ Assisstant Professor, Department of Orthopaedics, Dhanwantri Hospital and Research Centre, Jaipur. \\ 2 Professor, Department of Orthopaedics, Dhanwantri Hospital and Research Centre, Jaipur. \\ ${ }_{3}^{3}$ Assistant Professor, Department of Orthopaedics, SMS Medical College, Jaipur. \\ ${ }_{4}^{4}$ Resident, Department of Orthopaedics, Dhanwantri Hospital and Research Centre, Jaipur. \\ ${ }_{5}^{5}$ Resident, Department of Orthopaedics, Dhanwantri Hospital and Research Centre, Jaipur.
}

\section{ABSTRACT}

\section{OBJECTIVE}

Rotator cuff tears are a common shoulder problem and recently double-row repair techniques have shown superior biomechanical advantages when compared to single-row constructs in a properly selected patient. This study compared $2.8 \mathrm{~mm}$ and $4.5 \mathrm{~mm}$ anchors with respect to clinical and radiological outcome in two groups of patients. Of course, for the same number of 4.5 anchors used. Volume of Bone Replaced (VBR) will be far less if 2.8 anchors are used in equal number. But, in such surgeries, the ultimate objective was to restore the anatomic footprint and the number of anchors used varied accordingly.

\section{MATERIAL AND METHODS}

50 patients of rotator cuff tear (Size $>3 \mathrm{~cm}$ ) were treated with double-row method of repair arthroscopically. In 25 patients, 2.8 $\mathrm{mm}$ anchors were used and in rest 25 patients $4.5 \mathrm{~mm}$ anchors were used. Operated patients underwent CT scan of the shoulder during followup period. Using computer software (OsiriX), the cylindrical bone defects made for the anchors were measured by using the formula for volume of the cylinder. For every case, total Volume of Bone Replaced (VBR) was calculated. Since the plane of the anchor cannot always be sagittal or coronal, hence using MPR (Multiplanar reconstruction) tool in OsiriX, a plane was selected in which length of the anchor is the largest meaning that it is the plane parallel to true long axis of the anchor. Radius of the cylinder was calculated in a plane 90 degree to the previously defined plane.

\section{RESULTS}

Comparing the two groups, we didn't find any significant difference in the clinical outcome. However, there was a significant difference in one radiological parameter-Volume of Bone Replaced (VBR).

\section{CONCLUSION}

There was no difference in clinical recovery in the two groups. However, Volume of Bone Replaced (VBR) was more in $4.5 \mathrm{~mm}$ anchor group when compared to $2.8 \mathrm{~mm}$ anchor group. This finding can be important to a surgeon when he is operating a re-tear in an already operated double-row patient since there will be a relatively more bone stock available for the surgeon to place additional anchors.

\section{KEYWORDS}

Shoulder Arthroscopy; Rotator Cuff Tear; Double-Row Repair.

HOW TO CITE THIS ARTICLE: Saini O, Saini R, Meena UK, et al. Radiological and clinical assessment of two groups of anchors in double-row rotator cuff repair. J. Evolution Med. Dent. Sci. 2016;5(64):4524-4527, DOI: 10.14260/jemds/2016/1033

\section{INTRODUCTION}

Although, arthroscopic rotator cuff repair has been reported as a successful technique. Concerns still exist on failure rates of the procedure for large and massive cuff tears. ${ }^{1,2}$ In this paper, we aim to assess the volume of bone replaced while putting in anchors in double-row method of rotator cuff repair so as to have a quantitative data in mind when a future revision surgery is contemplated. We would be comparing volume of bone occupied by anchors while doing double-row repair using $4.5 \mathrm{~mm}$ anchors (in 25 subjects) and that while doing double-row repair using $2.8 \mathrm{~mm}$ anchors (in 25 subjects).

Financial or Other, Competing Interest: None.

Submission 19-04-2016, Peer Review 21-07-2016,

Acceptance 28-07-2016, Published 10-08-2016.

Corresponding Author:

Dr. Ojas Saini,

\#89-90, Sumer Nagar Vistar,

New Sanganer Road,

Mansarover, Jaipur-302020.

E-mail: ojassaini.kemortho@gmail.com

DOI: $10.14260 /$ jemds/2016/1033
Of course, for the same number of 4.5 anchors used, bone loss will be far less if 2.8 anchors are used in equal number. But, in such surgeries, the ultimate objective is to restore the anatomic footprint and the number of anchors used can vary accordingly. Hence, this study will also in a way show in what scenario will the volume of bone replaced be more when operating for massive cuff tears (cuff tear $>3 \mathrm{~cm}$ ) i.e. while using 2.8 anchors or while using 4.5 anchors. The two groups will also be compared for clinical outcome using constant shoulder score.

\section{MATERIALS AND METHODS Sample Size}

50 patients of rotator cuff tear were treated with Double-Row (DR) method of repair arthroscopically. (In 25 patients, 2.8 $\mathrm{mm}$ anchors have been used and in rest 25 patients $4.5 \mathrm{~mm}$ anchors have been used). Patient in this study were also evaluated using functional scores for clinical assessment. 


\section{Inclusion Criteria}

- Patients of rotator cuff tear treated with double-row method of arthroscopic repair technique within last 2 years at tertiary care centre in whom surgery was indicated for massive U-shaped cuff tear. Tear should at least be larger than $3 \mathrm{~cm}$.

- Those who give consent to be a part of this study.

\section{Exclusion Criteria}

- Patients with any other shoulder pathology including osteoarthritis of shoulder joint.

- $\quad$ Patients with history of fracture around shoulder joint in the past.

- Those in whom passive ROM of shoulder is severely restricted (e.g. Neglected stiff shoulder) as such cases will not allow true assessment of postop clinical outcome.

- Patients below 15 years of age group and those above 80 years of age group.

- Patient posted for revision arthroscopic shoulder cuff repair.

\section{Study Procedure}

Operated patients who met the inclusion criterion underwent a CT scan of the shoulder at 24 months postop follow up. Using computer software (OsiriX), the cylindrical bone defects made for the anchors have been measured by using the formula (Volume of the cylinder).

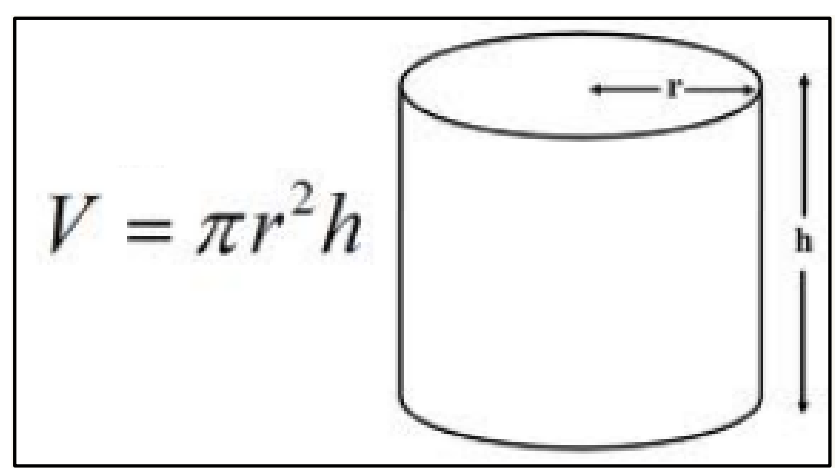

For every case, total Volumetric Bone Replaced (VBR) was calculated. Since, the plane of the anchor cannot always be sagittal or coronal, hence using MPR (Multiplanar reconstruction) tool in OsiriX, a plane has been selected in which length of the anchor is the largest meaning that it a plane parallel to true long axis of the anchor. Radius of the cylinder will be calculated in a plane 90 degrees to the previously defined plane (As this plane will show true axial cuts of the anchor and therefore radius can be calculated). All patients were operated upon by a senior arthroscopic surgeon. Institutional review board approval was obtained for this study.

\section{Surgery Protocol}

Patients underwent brachial plexus block and were placed in a lateral decubitus position. The arm was suspended at approximately $30^{\circ}-45^{\circ}$ of abduction and $20^{\circ}$ of forward flexion. Distraction of the shoulder joint was accomplished with traction.

\section{Technique for $\mathbf{2 . 8} \mathbf{~ m m}$ Anchor}

Inverted double-row technique: Place 2 inverted incline mattress stitches by turning suture passer upside down deep into the cuff for the medial-row fixation. Create the medial-row holes for the anchor implant $(2.8 \mathrm{~mm}$ anchor $)$ using appropriate bone punch at the articular margin of the footprint. Load each stitch into the implant and secure the bone lock once in the bone hole, then tension to desired cuff placement for restoration of the footprint contact area. Lock the stitch to implant tension with suture lock. Place 2 incline mattress stitches into the lateral aspect of the cuff for the lateral-row fixation. Create the lateral-row holes again using bone punch. Load each stitch into the anchor implant and secure the bone lock. Once in the bone hole, the tension the cuff to desired placement for restoration of the footprint contact area. Lock the stitch to implant after applying appropriate tension with suture lock.

\section{Technique for $4.5 \mathrm{~mm}$ Anchor}

Medial row is deployed again close to articular margin of the footprint using $4.5 \mathrm{~mm}$ anchors. The free ends of the sutures are passed through the torn edge of the rotator cuff to fashion a horizontal mattress stitch. Each pair of horizontal mattresses is tied and at this point the second suture from each anchor is cut as it will not be used for the final construct. The pair of sutures is then loaded through another $4.5 \mathrm{~mm}$ anchors, which are respectively used for lateral-row reconstruction.

\section{Postoperative Protocol}

- $\quad$ All the patients followed the same postoperative protocol.

- They wore a brace 24 hour a day with the operated shoulder at 15 degrees of abduction and in neutral rotation. During this early phase of initial two weeks, bracing was discontinued intermittently only for gentle joint oscillations in short-arm traction and various degrees of rotation with the arm in slight abduction.

- $\quad$ Suture removal was done after 2 weeks of surgery.

- Subsequently, a scheduled program of passive physical therapy 2 to 3 times a week was started. Only after a complete passive range of motion had been achieved active-assisted exercises and progressive muscle strengthening were begun. Patients returned to their normal activities of daily living 3 to 6 months after surgery.

At 24 months follow up, clinical evaluation was done in accordance with our case record form. Also, a CT scan was obtained for every patient. This CT scan was evaluated in OsiriX software to calculate volume of bone replaced while putting in anchors.

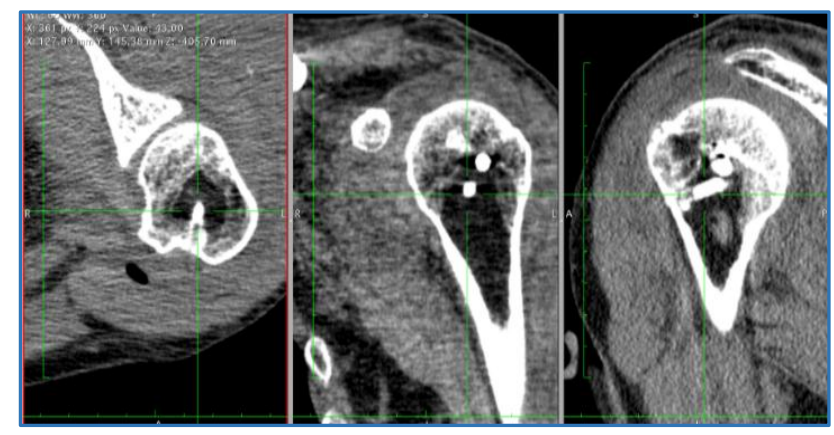

Fig. 1: Demonstrating Multiplanar Reconstruction Tool to Find Out Maximum Length and Breadth of the Anchor Hole 


\section{RESULTS AND OBSERVATIONS}

Statistical analyses was done using SPSS ver. 16 software. Paired $\mathrm{T}$ test and independent $\mathrm{T}$ test was used to find out whether the given finding was significant or not.

- Majority of patients (28) were between age group 45-60.

- Rotator cuff tear (Full thickness) are common in middle age group.

- Mean age: 58.58 Yrs.

- Majority of patients (68 percent) were male.

- In 32 (64\%) patients right-sided extremity was involved. There was no patient with bilateral involvement. Rightsided involvement was more common than left.

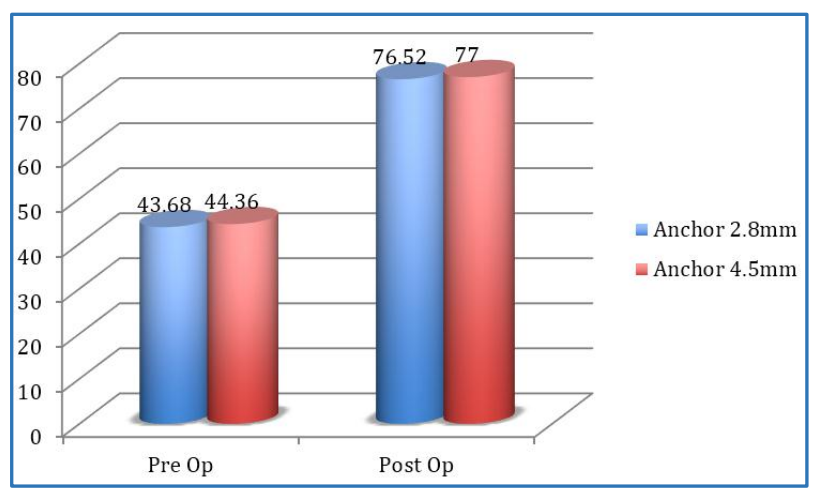

Chart 1

Chart 1: Demonstrating improvement in constant score (plotted on Y-axis) in postop patients in both $2.8 \mathrm{~mm}$ and 4.5 $\mathrm{mm}$ anchor group. There is statistically significant difference between preop and postop patients with respect to constant score within each group. But, when both the groups were compared, we found that the difference was statistically insignificant.

\section{Other Important Observations}

- Mean value of volume of bone replaced in $2.8 \mathrm{~mm}$ anchor group of patients: $372.470 \mathrm{~mm}^{3}$ (Standard deviation: $27.477 \mathrm{~mm}^{3}$ ).

- Mean value of volume of bone replaced in $4.5 \mathrm{~mm}$ anchor group of patients: $1182.664 \mathrm{~mm}^{3}$ (Standard deviation: $111.223 \mathrm{~mm}^{3}$ ).

- There is statistically significant difference between these two groups with respect to volume of bone replaced ( $p$ value $<0.05$ ).

\section{DISCUSSION}

Rotator cuff tears are a common shoulder problem. Using ultrasound, a prevalence of $20.7 \%$ was noted in the general population (With and without shoulder problems) by Yamamoto et al.(3) In a literature review of cadaver studies including 2553 shoulders from samples with a mean age of 70.1 years, prevalences of $11.8 \%$, and $18.5 \%$ were found for full thickness and partial thickness tears respectively.(4) Full thickness tears are rarely found in individuals under the age of 40 and tendon ageing is believed to be the most important contributing factor in the pathogenesis of rotator cuff tears. In our present study, only one patient aged below 45 years was diagnosed with the disease in question. Remaining 98 percent were aged above 45 years. Mean age was calculated to be around 58.58 years. Regarding sex incidence, study of literature reveals predominance of rotator cuff tears in males.
In our study, 68 percent of the entire sample size were males. Again, in our study, in 64 percent of the subjects, right side was affected. Maximising tendon healing is the primary goal of rotator cuff repair surgery. Traditional single-row techniques use one row of fixation points to secure the torn cuff to the humerus. Although, they have been clinically successful. They have been criticised for their inability to restore the medial-tolateral width of the cuff on the footprint.(5)A double-row repair secures the cuff using both medial and lateral anchors carries the theoretical advantage of reattaching the cuff to the whole of its footprint and therefore increases the contact area for healing. Studies in controlled laboratory environment have established that double-row techniques exhibit improved footprint repair with greater contact area and have an higher load to failure compared with single-row constructs in that environment. $(5,6,7)$ However, both randomised and nonrandomised clinical studies have not shown this biomechanical advantage to translate to superior clinical results when the two techniques were compared.(8,9) For comparing the two groups, the Constant-Murley score was used. The scoring system has been demonstrated to have high rates of intraobserver and interobserver correlation and to correlate strongly with patient satisfaction.(10) In our study which evaluated 50 patients undergoing double-row repair of rotator cuff tear $(>3 \mathrm{~cm})$, we found that there was an improvement in the constant score from 43.68 to 76.52 and from 44.36 to 77 in both the groups respectively (Refer chart 1 under 'Results and observations' ) after a mean followup of 24 months. But, between the two groups, our study didn't find any significant difference in the final clinical outcome. However, there was a significant difference in one radiological parameter between the two groups. Mean Volume of Bone Replaced (VBR) in $2.8 \mathrm{~mm}$ anchor group was $372.470 \mathrm{~mm}^{3}$ and mean VBR in $4.5 \mathrm{~mm}$ anchor group was $1182.664 \mathrm{~mm}^{3}$.We propose that this fact can be important to a surgeon when he is operating a re-tear in already operated double-row patient since there will be a relatively more bone stock available for the surgeon to place additional anchors. Moreover, in our study, we didn't find any significant difference in the clinical outcome while using $2.8 \mathrm{~mm}$ anchors both in terms of overall constant score and in terms of overall improvement in range. Furthermore, issue of re-tear is important as various studies done for large and massive tears have reported $17 \%$ to $44 \%$ re-tear rates.(11,12,13,14) The study had several limitations. The sample size was less. Each group had 25 patients from which we could not derive firm conclusions. Minimal errors in calculation of radiological parameters due to beam hardening artefact cannot be ruled out either.

\section{CONCLUSION}

In this study, fifty patients with large rotator cuff tears $(>3 \mathrm{~cm})$ operated with double-row arthroscopic technique were analysed. The functional outcome was calculated at the end of six months after surgery with help of Constant-Murley score. Mean age of the population of study group was 58.58 year. Comparing two groups $(2.8 \mathrm{~mm}$ anchor group and $4.5 \mathrm{~mm}$ anchor group), we didn't find any significant difference in the clinical outcome both in terms of overall constant score and in terms of overall improvement in range of shoulder motion. However, there was a significant difference in one radiological parameter between the two groups - Volume of Bone Replaced (VBR). This finding can be important to a surgeon when he is 
operating a re-tear in already operated double-row patient since there will be a relatively more bone stock available for the surgeon to place additional anchors.

\section{ACKNOWLEDGEMENT}

All the authors have read and approved the final manuscript. No conflicts of interest were declared by the authors. No external funding was received for this study.

\section{REFERENCES}

1. Bishop J, Klepps S, Lo IK, et al. Cuff integrity after arthroscopic versus open rotator cuff repair: a prospective study. J Shoulder Elbow Surg 2006;15(3):290-9.

2. Galatz LM, Ball CM, Teefey SA, et al. The outcome and repair integrity of completely arthroscopically repaired large and massive rotator cuff tears. J Bone Joint Surg Am 2004;86-A(2):219-24.

3. Yamamoto A, Takagishi K, Osawa T, et al. Prevalence and risk factors of a rotator cuff tear in the general population. J Shoulder Elbow Surg 2010;19(1):116-20.

4. Reilly P, Macleod I, Macfarlane R, et al. Dead men and radiologists don't lie: a review of cadaveric and radiological studies of rotator cuff tear prevalence. Ann $\mathrm{R}$ Coll Surg Engl 2006;88(2):116-21.

5. Lo IKY, Burkhart SS. Double-row arthroscopic rotator cuff repair: re-establishing the footprint of the rotator cuff. Arthroscopy 2003;19(9):1035-42.

6. Meier SW, Meier JD. Rotator cuff repair: the effect of double-row fixation on three-dimensional repair site. J Shoulder Elbow Surg 2006;15(6):691-6.

7. Kim DH, Elattrache NS, Tibone JE, et al. Biomechanical comparison of a single-row versus double-row suture anchor technique for rotator cuff repair. Am J Sports Med 2006;34(3):407-14.
8. Franceschi F, Ruzzini L, Longo UG, etal. Equivalent clinical results of arthroscopic single-row and double-row suture anchor repair for rotator cuff tears: a randomised controlled trial. Am J Sports Med 2007;35(8):1254-60.

9. Charousset C, Grimberg J, Duranthon LD, et al. Can a double-row anchorage technique improve tendon healing in arthroscopic rotator cuff repair? A prospective, nonrandomised, comparative study of double-row and single-row anchorage techniques with computed tomographic arthrography tendon healing assessment. Am J Sports Med 2007;35(8):1247-53.

10. Johansson KM, Adolfsson LE. Intraobserver and interobserver reliability for the strength test in the Constant-Murley shoulder assessment. J Shoulder Elbow Surg 2005;14(3):273-8.

11. Lafosse L, Brozska R, Toussaint B, et al. The outcome and structural integrity of arthroscopic rotator cuff repair with use of the double-row suture anchor technique. Journal Bone Joint Surgery 2007;89(7):1533-41.

12. Huijsmans PE, Pritchard MP, Berghs BM, et al. Arthroscopic rotator cuff repair with double-row fixation. Journal Bone Joint Surgery Am 2007;89(6):1248-57.

13. Sugaya H, Maeda K, Matsuki K, et al. Repair integrity and functional outcome after arthroscopic double-row rotator cuff repair: a prospective outcome study. Journal Bone Joint Surgery Am 2007;89(5):953-60.

14. Sugaya $H$, Maeda $K$, Matsuki $K$, et al. Functional and structural outcome after arthroscopic full-thickness rotator cuff repair: single-row versus dual-row fixation. Arthroscopy 2005;21(11):1307-16. 